\title{
Tracheostomy Tube Blockage with an Unusual Foreign Body- A Reminder of the Importance of the Tracheostomy Emergency Management Algorithm
}

\author{
Tom Bradish, MBBS, MRCS (ENT) and Muhammad Shakeel, MBBS, B.SC, MRCSED, DOHNS, \\ FRCS (ORL-HNS)
}

Department of Otolaryngology-Head and Neck Surgery, Aberdeen Royal Infirmary, Aberdeen, UK

\begin{abstract}
Introduction: Patients with tracheostomies are common. Despite this many healthcare providers are uncomfortable managing tracheostomy emergencies despite the fact that they are common critical airway events. Head and neck patients are also at risk of multifactorial delirium which can lead to or exacerbate tracheostomy issues.

Aims: To highlight the importance National tracheostomy safety project emergency algorithm. To raise awareness of causes of delirium in head and neck patients.

Case report: We present a case of a tracheostomy tube blocked with a cap from a feeding gastrostomy set resulting in a difficult to spot complete tracheostomy occlusion. We discuss the importance of multidisciplinary approach to reduce risk of delirium in head and neck cancer patients.
\end{abstract}

Conclusion: All healthcare providers should be aware of the emergency tracheostomy management algorithm. Extra steps must be taken in head and neck patients to help avoid delirium.

\section{Introduction}

Every healthcare professional will encounter a patient with a tracheostomy at some point in their career. Common reasons for tracheostomy include upper airway obstruction, ventilatory wean, secretion management, aspiration management. During the EPIC study of intensive care units in $1997,16 \%$ of all ICU patients in the UK had a tracheostomy [1]. In 2009/12 over 10700 tracheostomies (surgical and percutaneous) were performed in the UK [2]. Despite their relative abundance many clinicians are unaware of how to approach assessing the airway of a tracheostomy patient. The McGrath and Thomas review of cases reported to the National Patient Safety Agency found that $60 \%$ of critical airway events in which patients were directly affected were related to displaced or blocked tracheostomy tubes [3].

As a result of the above findings, the national tracheostomy safety project was founded to improve the management of tracheostomy and laryngectomy critical incidents. Despite this, many clinicians are not comfortable or aware of the emergency algorithm for tracheostomy management (Figure 1) [2].

We present a case of a blocked tracheostomy as a learning point to raise awareness of the tracheostomy emergency management algorithm but also to highlight a possible patient safety concern regarding patients with tracheostomy and feeding gastrostomy tube.

\section{Case Presentation}

A 56-year-old male with a radio recurrent inoperable tongue base squamous cell carcinoma underwent palliative tracheostomy due to intermittent airway obstruction and radiologically inserted feeding gastrostomy due to aspiration. Following a period of training on the ward he was discharged home with palliative care with a size 8 fenestrated uncuffed Shiley ${ }^{\mathrm{TM}}$ tracheostomy tube in place. His feeding gastrostomy was compatible with Nutricia Flocare ${ }^{\circledR}$ Infinity ${ }^{\mathrm{TM}}$ feeding tubes and pumps.

1 month later he was found in the morning unresponsive by his carers and transferred to hospital by emergency

*Corresponding author: Muhammad Shakeel, Department of Otolaryngology-Head and Neck Surgery, Aberdeen Royal Infirmary, Aberdeen, AB25 2ZN, Scotland, UK

Accepted: February 12, 2022

Published online: February 14, 2022

Citation: Bradish T, Shakeel M (2022) Tracheostomy Tube Blockage with an Unusual Foreign Body- A Reminder of the Importance of the Tracheostomy Emergency Management Algorithm. J Head Neck Surg 5(1):176-179 


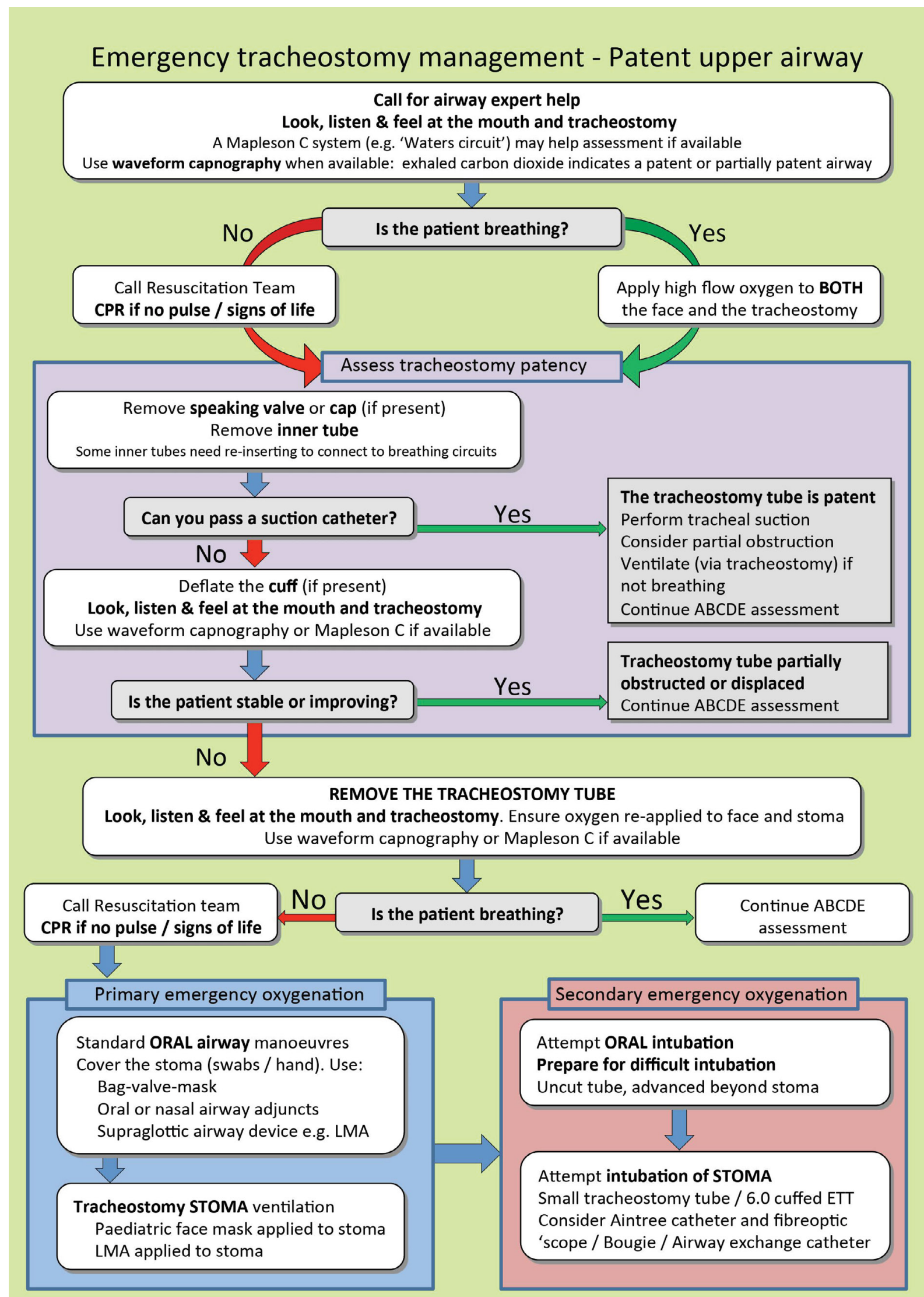

National Tracheostomy Safety Project. Review date 1/4/16. Feedback \& resources at www.tracheostomy.org.uk

Figure 1: Emergency tracheostomy mangement algorithm, reproduced with permission from the National Tracheostomy Safety Project. 

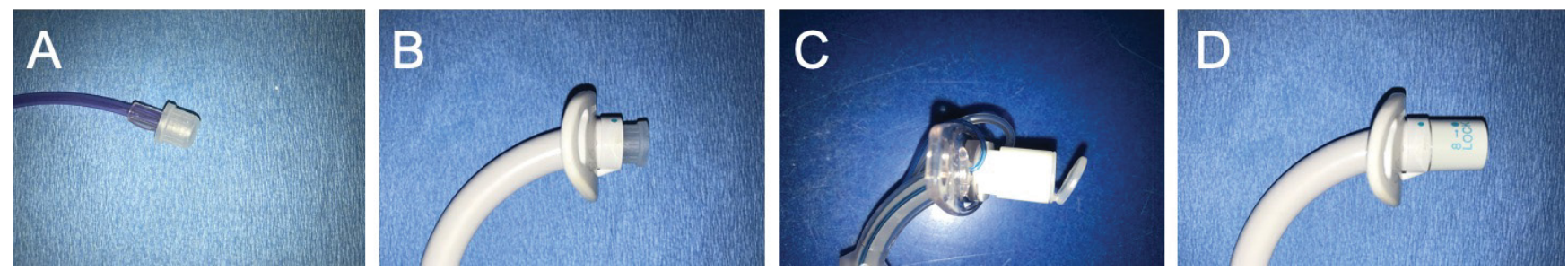

Figure 2: A) The disposable feeding cap on a Nutricia Flocare ${ }^{\circledR}$ Infinity ${ }^{\mathrm{TM}}$ feeding tube; B) The cap in situ in a Shiley ${ }^{\mathrm{TM}}$ tracheostomy tube creating an occlusive cap and airway obstruction; C) Portex ${ }^{\mathrm{TM}}$ tracheostomy tube with inner cannula in situ which has an appearance similar to B; D) The correct appearance of a Shiley ${ }^{\mathrm{TM}}$ tracheostomy tube with inner cannula.

ambulance with ENT/A+E prealert. During ambulance transfer supplemental oxygen + nebulisation via mouth and tracheostomy was given and the patient became more responsive and agitated with peripheral oxygen saturations of $84 \%$. Blood pressure was $64 / 46$, temperature $36.5^{\circ} \mathrm{C}$.

On arrival in Accident and Emergency the tracheostomy tube was inspected and found to be blocked with the disposable cap from the Nutricia Flocare ${ }^{\circledR}$ Infinity ${ }^{\mathrm{TM}}$ feeding tube. This cap (Figure 2A) happens to fit into all sizes of Shiley ${ }^{\mathrm{TM}}$ tracheostomy tube (when no inner cannula is in situ) to result in difficult to spot occlusive cap (Figure 2B). The cap was removed, tracheostomy tube placement patency was confirmed with flexible endoscopy and a new inner cannula was inserted.

The patient returned to GCS 15; blood pressure improved the patient was admitted to the ward. Bloods on admission showed WCC 19.1, Neutrophils 17.5 which was due to aspiration pneumonia. Patient medications on admission included Long acting morphine sulphate, Pregabalin, Dexamethasone, Amitriptyline, PRN Liquid oral morphine, PRN Lorazepam alongside paracetamol, furosemide, ibuprofen, laxido and omeprazole. The patient was treated for aspiration pneumonia and underwent palliative hospice discharge 1 week later. A yellow card was filled out to report this as a possible event using a medical device.

\section{Discussion}

Tracheostomy tubes vary in appearance and every brand has a different appearance to the inner cannula. To a healthcare professional not dealing with tracheostomy tubes on a regular basis the appearance of the Shiley ${ }^{\mathrm{TM}}$ tube with no inner cannula blocked with the feeding gastrostomy cap looks remarkably similar to a Portex tracheostomy tube with an inner cannula (Figure 2C). In this patient, if the national tracheostomy safety project algorithm (Figure 1) had been followed the obstruction would have been identified and potentially cleared much sooner.

This patient improved with application of oxygen to both the face and the neck. This demonstrates the importance of considering some upper airway patency in tracheostomy patients. In this patient this supplemental oxygen via facemask was possibly the difference between alive or dead on arrival in $\mathrm{A}+\mathrm{E}$ given the 50 minutes ambulance drive from hospital.

Head and Neck cancer patients are at risk of multifactorial delirium. They are more likely to be on multiple medications, a known independent risk factor for delirium [4]. They are more prone to metabolic disturbances, aspiration events and subsequent infections. They are more prone to malnutrition and dehydration [5] as well as sleep deprivation due to pain or medications. Given the risk profile of a head and neck cancer patients they are also more likely to be prone to alcohol intoxication [6]. On questioning our patient could not remember putting the feeding tube cap on his tracheostomy tube so the exact circumstances are unknown. It is known that even conscious patients cannot always be relied on for an accurate foreign body history [7]. The fact that this event is a possible occurrence needs addressing. One approach is addressing the cause of possible delirium. Any head and neck cancer patient should have medications rationalised to limit polypharmacy and co-prescribing of medications known to cause delirium (e.g., analgesics, sedatives, antihistamines, steroids). The presence of Dieticians and Speech and language therapists on the head and neck MDT is vital to identify feeding problems and patients at risk of aspiration and malnutrition. GP colleagues and alcohol cessation services should be used to try to address any intoxication which would affect tracheostomy care.

However, the authors feel that the near miss event involving the gastrostomy cap should be addressed so that it cannot happen again. The authors feel that the shape of the cap should be changed to mean that it does not fit and stay in the Shiley ${ }^{\mathrm{TM}}$ tube.

\section{Conclusion}

Every medical professional should be aware of the national tracheostomy project emergency management algorithm as this is the easiest and best was of assessing tracheostomy tube patency. Initial management should include use of suction catheter to determine tracheostomy patency as well as application of oxygen to both the face and the neck. Medical professionals should be aware that head and neck cancer patients are at increased risk of delirium and consider this in their management.

\section{Acknowledgements}

Emergency tracheostomy management algorithm reproduced with permission of National Tracheostomy Safety Project.

\section{Financial Support}

This research received no specific grant from any funding agency, commercial or not-for-profit sectors. 


\section{Conflict of Interest}

None declared by all authors.

\section{References}

1. Vincent JL, Suter P, Bihari D, et al. (1997) Organization of intensive care units in Europe: Lessons from the EPIC study. Intensive Care Med 23: 1181-1184.

2. McGrath BA, Bates L, Atkinson D, et al. (2012) Multidisciplinary guidelines for the management of tracheostomy and laryngectomy airway emergencies. Anaesthesia 67: 1025-1041.

3. Thomas AN, McGrath BA (2009) Patient safety incidents associated with airway devices in critical care: A review of reports to the UK national patient safety agency. Anaesthesia 64: 358-365.
4. Hein C, Forgues A, Piau A, et al. (2014) Impact of polypharmacy on occurrence of delirium in elderly emergency patients. J Am Med Dir Assoc 15: 850.e11-850.e15.

5. Yanni A, Dequanter D, Lechien JR, et al. (2019) Malnutrition in head and neck cancer patients: Impacts and indications of a prophylactic percutaneous endoscopic gastrostomy. Eur Ann Otorhinolaryngol Head Neck Dis 136: S27-S33.

6. Kawakita D, Matsuo K (2017) Alcohol and head and neck cancer. Cancer Metastasis Rev 36: 425-434.

7. Sankar V, Shakeel M, Keh S, et al. (2012) A case of a "lost" nasogastric tube. J Laryngol Otol 126: 1296-1298.

DOI: $10.36959 / 605 / 565$

Copyright: (C) 2022 Bradish T, et al. This is an open-access article distributed under the terms of the Creative Commons Attribution License, which permits unrestricted use, distribution, and reproduction in any medium, provided the original author and source are credited. 